\title{
CARBONO ORGÁNICO DEL SUELO EN DIFERENTES USOS DEL TERRENO DE PAISAJES ANDINOS COLOMBIANOS
}

\section{Soil Organic Carbon in Different Land Uses of Colombian Andean Landscapes}

\author{
Andrés F. Carvajal ${ }^{*}$, Alexander Feijoo ${ }^{1}$, Heimar Quintero ${ }^{2}$, Marco A. Rondón $^{3}$ \\ ${ }^{1}$ Universidad Tecnológica de Pereira, Facultad de Ciencias Ambientales, Grupo Gestión en \\ Agroecosistemas Tropicales Andinos (GATA), Vereda La Julita, Pereira, Colombia. ${ }^{2}$ Profesor \\ pensionado Universidad Nacional de Colombia Sede Palmira, Valle del Cauca, Colombia. \\ ${ }^{3}$ International Development Research Centre (IDRC), Ottawa, Ontario, Canadá. * Corresponding \\ author: acarvaja@utp.edu.co
}

\begin{abstract}
The relationship between changes in land use and soil carbon and nitrogen was assessed in Alcalá (colombian coffee growing region), between 900 and $1600 \mathrm{~m}$. Soils are Argiudolls and Melanudands and sampling units were defined according to the altitudinal gradient. Carbon and nitrogen content, $\mathrm{C}: \mathrm{N}$ ratio, bulk density and $13 \mathrm{C}$ and ${ }^{15} \mathrm{~N}$ abundances were measured. Significant differences $(\mathrm{p}<0.05)$ were found due to altitude effect, differentiating the high zone by high $\mathrm{C}\left(37.06-42.88 \mathrm{t} \mathrm{ha}^{-1}\right), \mathrm{N}(3.12-$ $\left.3.86 \mathrm{t} \mathrm{ha}^{-1}\right)$ and $\mathrm{C}: \mathrm{N}(11.17-11.97)$, low bulk density $\left(0.56-0.65 \mathrm{~g} \mathrm{~cm}^{-3}\right)$ and ${ }^{15} \mathrm{~N}$ abundance (4.59-5.15\%). Fallow and monocultures stored more carbon in deeper layers of the soil, while most preserved sites showed low $\mathrm{C}, \mathrm{N}$ contents. The ${ }^{13} \mathrm{C}$ in land uses ranged between $-25.18 \%$ and $-21.17 \%$, indicating that carbon was fixed mainly by $\mathrm{C}_{3}$ plants. It was concluded that land use changes affect $\mathrm{C}$ stocks, due to practices like chemical fertilization; and Alcalá soils have high potential for ecosystem services related with edaphic carbon capture.
\end{abstract}

Keywords: carbon sequestration in the Andes, land use changes, stable isotopes

\section{RESUMEN}

Se estudió la relación de cambios en el uso del terreno con el $\mathrm{C}$ y $\mathrm{N}$ edáficos, en el municipio de Alcalá (región cafetera colombiana), entre 900 y 1600 m de elevación. Los suelos son Argiudolls y Melanudands y las unidades de muestreo fueron definidas según el gradiente altitudinal. Se midieron contenidos de $\mathrm{C}$ y $\mathrm{N}$, relación $\mathrm{C}: \mathrm{N}$, densidad aparente y abundancias de ${ }^{13} \mathrm{C} y{ }^{15} \mathrm{~N}$. Se encontraron diferencias significativas $(\mathrm{p}<0,05)$ por el efecto de la altura. La zona alta mostró mayores contenidos de $\mathrm{C}(37,06-42,88 \mathrm{t}$ $\left.\mathrm{ha}^{-1}\right)$ y $\mathrm{N}\left(3,12\right.$ - 3,86 $\left.\mathrm{t} \mathrm{ha}^{-1}\right)$, mayor relación C:N (11,17 - 11,97), menor densidad aparente $\left(0.56-0.65 \mathrm{~g} \mathrm{~cm}^{-3}\right)$ y abundancia de $15 \mathrm{~N}(4,59-5,15 \%)$. Barbechos $\mathrm{y}$ monocultivos almacenaron más carbono en la parte subterránea del suelo; mientras que en sitios más conservados los contenidos de $\mathrm{C}$ y $\mathrm{N}$ fueron bajos. $\mathrm{El}{ }^{13} \mathrm{C}$ según los usos, fluctuó entre $-25,18$ y $-21,27 \%$ indicando que la mayoría del $\mathrm{C}$ fue fijado por plantas $\mathrm{C}_{3}$. Se concluyó que los cambios en el uso afectan el almacenamiento de $\mathrm{C}$, 
principalmente por prácticas como fertilización con productos de síntesis química; y que los suelos de Alcalá tienen alto potencial para la prestación de servicios ecosistémicos relacionados con la captura de carbono edáfico.

Palabras clave: Almacenamiento de carbono en los Andes, cambios en el uso del terreno, isótopos estables.

\section{INTRODUCCIÓN}

Las concentraciones atmosféricas de $\mathrm{CO}_{2}$, $\mathrm{CH}_{4}$ y $\mathrm{N}_{2} \mathrm{O}$ se están incrementando por actividades antrópicas como el cambio en los usos del terreno. Esto ha provocado una tasa de calentamiento global que probablemente excede la tasa crítica en la que los ecosistemas son capaces de adaptarse a cambios climáticos (IPCC, 2007; Lal, 2004). Globalmente los suelos a un metro de profundidad contienen 1500 Gt de carbono, de las que se estima que el $44 \%$ está en los trópicos, donde los tiempos de residencia de la materia orgánica son menores y por lo tanto los depósitos de $\mathrm{C}$ son más susceptibles a la sustitución de usos (López-Ulloa, 2005). De allí que las pérdidas de este elemento se den por la conversión de ecosistemas naturales a sistemas agrícolas por la reducción en los aportes de materia orgánica y la protección física del suelo (Tan y Lal, 2005).

En Colombia en el año 2003 el café fue el primer producto agrícola en extensión con 775.000 ha, representando el $20 \%$ del área cultivada en este país y el $34 \%$ de los cultivos permanentes (Espinal et al., 2005). En el Eje Cafetero colombiano ubicado en el CentroOccidente del territorio, la modernización de los sistemas de producción aceleró la sustitución de selvas Andinas por monocultivos y potreros; ocasionando pérdida de cobertura vegetal natural.

En el municipio de Alcalá, gran parte de los sistemas finca se transformó entre 1960 y 1990 siguiendo políticas gubernamentales e institucionales que promovían los monocultivos de café (Zúñiga et al., 2003); aunque algunos agricultores conservaron variedades con sombrío (Feijoo et al., 2007) y mantuvieron zonas de conservación con relictos de selva, barbechos y guaduales para la protección de fuentes hídricas; haciendo que el paisaje del municipio fuera heterogéneo, con historia de usos, coberturas y manejo variables.

En los Andes colombianos poco se han evaluado almacenamientos de carbono de acuerdo con las variaciones en el paisaje, lo que es de gran importancia porque este elemento depende de condiciones específicas de cada sitio, requiriendo métodos de muestreo que permitan representar cambios a nivel local y del paisaje para la identificación de patrones de distribución (Leifeld et al., 2005). Por otra parte, Batjes (2005) plantea que los agroecosistemas pueden ser manejados para reducir emisiones e incrementar depósitos de $\mathrm{C}$, pero las opciones se deben escoger con base en el conocimiento de la magnitud de los almacenamientos edáficos de un bioma o región agroecológica, y la respuesta de los suelos a diferentes usos.

Por lo anterior, este trabajo propuso evaluar las variaciones en los almacenamientos de $\mathrm{C}$ y $\mathrm{N}$ en diferentes usos del terreno, localizados en tres zonas altitudinales del municipio de Alcalá, Valle del Cauca; y planteó como hipótesis que los suelos de esta región subandina tienen alta capacidad para la captura de estos dos elementos, la cual es afectada 
por la altura sobre el nivel del mar y por los cambios en el uso del terreno, dependiendo no solo de la cobertura vegetal actual, sino además de sistemas anteriores que han contribuido con la fijación.

\section{MATERIALES Y MÉTODOS}

\section{Localización del estudio}

El estudio se adelantó en el municipio de Alcalá, Valle del Cauca, Colombia, en la vertiente occidental de la Cordillera Central entre las coordenadas $75^{\circ} 51^{\prime} 29^{\prime}$ ' $\mathrm{W}, 4^{\circ} 43^{\prime} 8^{\prime}$ ' $\mathrm{N}$ y $75^{\circ} 42^{\prime} 18^{\prime}$ ' $\mathrm{W}, 4^{\circ} 39^{\prime}$ 0 ', con temperatura de $18-24^{\circ} \mathrm{C}$, precipitación anual de $1350-2400 \mathrm{~mm} \mathrm{y}$ humedad relativa entre $65-80 \%$. El clima en la zona es bimodal con dos temporadas secas (diciembre-febrero y junio-agosto) y dos lluviosas (marzo-mayo y septiembrenoviembre) (Alcaldía municipal de Alcalá, 2003).

Se consideró como universo poblacional el área del municipio entre 1150 y 1600 msnm, para la cual se definieron tres estratos con rangos de 150 m. En cada estrato se construyeron dos ventanas conformadas por 16 puntos de muestreo distribuidos en una red cuadrada con espacios de $200 \mathrm{~m}$ entre puntos de muestreo. La ventana uno y dos se localizaron en las veredas La Cuchilla, El Congal y Maravélez; la tres y cuatro en la en La Polonia y Bélgica; y la cinco y seis en El Higuerón (Figura 1).

Los suelos pertenecen a las unidades Malabar y Chinchiná, ambas provenientes de cenizas volcánicas pero con diferencias en la textura, saturación de bases, fertilidad, estructura y drenaje interno (Alcaldía municipal de Alcalá, 2003; Cenicafé, 2007) (Cuadro 1). El paisaje en cada una de las ventanas fue heterogéneo, encontrándose en total 18 usos del terreno, predominando el pastizal
(Cynodon nlemfuensis) en la zona baja (12 muestreos); café-plátano (Coffea arabica L-Musa paradisiaca) (8) y monocultivo de café variedad Colombia (Coffea arabica L., cv. Colombia) (6) en la media; y café variedad Colombia (7) y relicto de selva (6) en la alta.

Los monocultivos de café y la mayoría de cafetales asociados con otras especies vegetales recibieron adiciones de fertilizantes de síntesis química como urea, 15-15-15 y 25-4-24, en cantidades que variaron entre 20 y $300 \mathrm{~g} /$ planta/año. Además se podaron cada cuatro o cinco años y la frecuencia de eliminación de arvenses estuvo entre una y tres veces por año. En pastizales, relictos de selva $\mathrm{y}$ barbechos no se realizaron aplicaciones de insumos, mientras que en algunos guaduales (Guadua angustifolia Kunth) se realizó extracción de material de forma esporádica.

En relación con la historia de los usos del terreno en el área de estudio, los sitios que actualmente se encuentran en cultivos de café asociados y en monocultivo, anteriormente fueron selvas nativas, mientras los pastizales han tenido un proceso más largo, porque posterior a la selva nativa, se encontraron cultivos de café que finalmente se convirtieron en potreros.

\section{Almacenamiento de carbono y nitrógeno en el suelo}

Para la estimación del contenido de carbono y nitrógeno en el suelo se realizó un muestreo sistemático estratificado (método de la red cuadrada) (Cochran, 1974). Se realizaron 96 monolitos $(0.25 \mathrm{x}$ $0.25 \times 0.3 \mathrm{~m})$ en los que se tomaron muestras de suelo en 0-10, 10-20 y 20-30 $\mathrm{cm}$ de profundidad, las cuales se analizaron por medio de combustión seca a alta temperatura (analizador $\mathrm{CHN}$ Europa Scientific), en el laboratorio de la Universidad de Davis, California, Estados 
Unidos donde también se determinó la abundancia isotopica natural de ${ }^{13} \mathrm{C} \mathrm{y}{ }^{15} \mathrm{~N}$. En cada profundidad se tomaron muestras con un cilindro metálico $\left(98,12 \mathrm{~cm}^{3}\right)$. Se registró el peso húmedo (P1), se secaron las muestras de suelo y se utilizó el peso seco (P2) en la determinación la densidad aparente (DA) con la fórmula:

$$
\mathrm{DA}\left(\mathrm{g} / \mathrm{cm}^{3}\right)=\frac{(\mathrm{P} 1-\mathrm{P} 2)}{\text { Volumen interno del cilindro }}
$$

Los contenidos de carbono y nitrógeno se calcularon de la siguiente manera:

Carbono en suelo $\left(\mathrm{t} \mathrm{ha}^{-1}\right)=\% \mathrm{C} * \mathrm{DA} * \mathrm{E}^{*} \mathrm{~A} * 100$ Nitrógeno en suelo $\left(\mathrm{t} \mathrm{ha}^{-1}\right)=\% \mathrm{~N} * \mathrm{DA} * \mathrm{E}^{*} \mathrm{~A} * 100$

Donde: $\% \mathrm{C}=$ Porcentaje de carbono; $\% \mathrm{~N}=$ Porcentaje de nitrógeno; DA $=$ Densidad aparente $\left(\mathrm{g} / \mathrm{cm}^{3}\right) ; \mathrm{E}=$ Espesor de la capa de muestreo (cm); A = Área considerada (1 ha).

\section{Abundancia natural de ${ }^{13} \mathrm{C} y{ }^{15} \mathrm{~N}$}

Se calcularon las abundancias isotópicas naturales de ${ }^{13} \mathrm{C}$ y ${ }^{15} \mathrm{~N}$ con base en los valores estándares del V-PDB (Viena-Pee Dee Belemnite) y el nitrógeno del aire (Peterson y Fry, 1987); utilizando las siguientes fórmulas;

$$
\begin{aligned}
\delta^{13} C & =\left(\frac{\left({ }^{13} C /{ }^{12} C\right) \text { muestra }}{\left({ }^{13} C /{ }^{12} C\right) V P D B}-1\right) \times 1000 \\
\delta^{15} N & =\left(\frac{\left({ }^{15} N /{ }^{14} N\right) \text { muestra }}{\left({ }^{15} N / I^{14} N\right) \text { Aire }}-1\right) \times 1000
\end{aligned}
$$

\section{Análisis de la información}

Para las variables fisicoquímicas del suelo (densidad aparente, $\% \mathrm{C}, \quad \% \mathrm{~N}$, almacenamientos de $\mathrm{C}$ y $\mathrm{N}$, relación $\mathrm{C}: \mathrm{N}$,

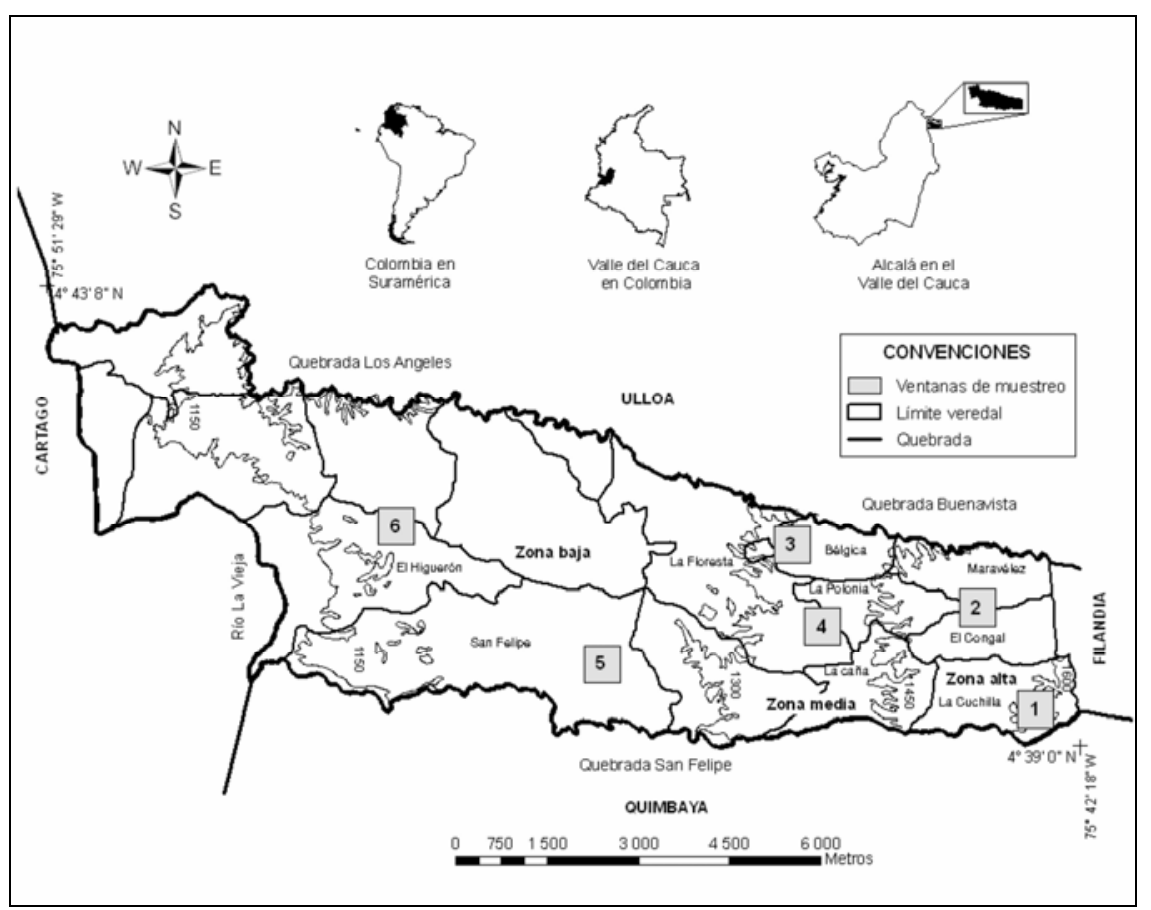

Figura 1. Localización de las ventanas de muestreo en tres zonas altitudinales del municipio de Alcalá.

Figure 1. Location of sampling windows in three altitudinal zones of Alcalá municipality. 
Cuadro 1. Características de los suelos de la zona de estudio

Table 1. Soil characteristics of study area

\begin{tabular}{lcc}
\hline Parámetro & Unidad Malabar & Unidad Chinchiná \\
\hline $\begin{array}{l}\text { Orden del suelo } \\
\text { Clasificación } \\
\text { taxonómica }\end{array}$ & Alfisol & Andisol \\
Rango altitudinal (m) & Argiudolls Endoaqualfs & Melanudands Fulvudands \\
Origen & & Hapludands \\
Textura & $900-1250$ & $1250-1600$ \\
Fertilidad & Cenizas volcánicas & Cenizas volcánicas \\
Saturación de bases & Franco arcilloso- Arcilloso & Franco \\
Capacidad & Media-Alta & Baja \\
intercambio catiónico & $40-50 \%$ & $20-35 \%$ \\
Materia orgánica & $18-20$ cmol kg-1 & $11-30$ cmol kg ${ }^{-1}$ \\
Color & & \\
& $1-10 \%$ & $1-15 \%$ \\
Tipo estructura & Pardo oscuro a & Negro a pardo amarillento \\
& pardo amarillento oscuro & Blocosa angular y subangular \\
Clase estructura & Blocosa subangular & \\
Grado estructura & y migajosa & Fina \\
Drenaje interno & Mediana a fina & Moderada a fuerte \\
Drenaje externo & Moderada a fuerte & Rápido \\
Presencia de poros & Lento & Rápido \\
pH & Rápido & Abundantes y finos \\
\hline
\end{tabular}

${ }^{13} \mathrm{C}$ y ${ }^{15} \mathrm{~N}$ ) se realizaron pruebas de Kruskal Wallis para muestras independientes con el programa SPSS versión 10.0 (SPSS Inc, 1999), para buscar diferencias estadísticamente significativas $(\mathrm{p}<0,05)$ entre zonas de muestreo y entre profundidades del suelo.

Se realizó Análisis de Componentes Principales (ACP) para reducir la dimensión de los datos, agrupando variables y facilitando la explicación de la variabilidad total con los dos primeros componentes extraídos. Se incluyeron las variables $\% \mathrm{C}, \% \mathrm{~N}$, almacenamiento de $\mathrm{C}$, almacenamiento de $\mathrm{N}$, relación $\mathrm{C}: \mathrm{N}$, densidad aparente, ${ }^{13} \mathrm{C}$ y ${ }^{15} \mathrm{~N}$ y se utilizó como categoría de agrupación el uso del terreno, para posteriormente realizar análisis discriminante y buscar diferencias significativas entre estos grupos. En el análisis estadístico se utilizó el programa $\mathrm{R}$ versión 2.7.1 ( $\mathrm{R}$ Development Core Team, 2008).

\section{RESULTADOS Y DISCUSIÓN}

La profundidad del suelo influyó sobre los contenidos de materia orgánica, encontrando más $\mathrm{C}$ y $\mathrm{N}$ almacenado en la capa superficial con tendencia a disminuir hacia las capas inferiores, presentando diferencias altamente significativas $(\mathrm{p}<0,05)$ entre $0-10,10-20$ y $20-30 \mathrm{~cm}$. $15 \mathrm{~N}$ presentaron los valores más altos en 20-30 cm (Cuadro 2). Las diferencias entre 
0-10 y 20-30 cm para los contenidos de C, fueron de $5.8 \mathrm{t} \mathrm{ha}^{-1}$ en la zona alta, $10.4 \mathrm{t}$ $\mathrm{ha}^{-1}$ en la media y $3 \mathrm{t} \mathrm{ha}^{-1}$ en la baja. En relación con los cambios en la profundidad, Diekow et al. (2005) en Brasil encontraron una tendencia diferente, donde cultivos con asociaciones de cereales y leguminosas almacenaron mayores cantidades de carbono a partir de $17,5 \mathrm{~cm}$, lo que explicaron por la posible disposición de material orgánico por medio del aporte directo de las raíces o por el transporte de residuos orgánicos a través del perfil por la meso y macrofauna.

Contrariamente las abundancias naturales de los isótopos estables aumentaron con la profundidad. Krull y Skjemstad (2003) en Australia y Michelsen et al. (2004) en Etiopía encontraron que el ${ }^{13} \mathrm{C}$ incrementó desde la superficie hacia las partes más profundas del suelo, lo que es consecuente con mayores pérdidas respiratorias de carbono isotópicamente ligero durante la descomposición de las capas superficiales del suelo, resultando en enriquecimiento del carbono más antiguo en profundidad.

Igualmente, los valores de $\delta^{15} \mathrm{~N}$ incrementaron hacia $20-30 \mathrm{~cm}$, tendencia también reportada por Eshetu (2004) que mostró que el ${ }^{15} \mathrm{~N}$ a pesar de no ser sensible al tipo fotosintético de las plantas, estuvo en función de los procesos de mineralización al interior del suelo (Boeckx et al., 2006). De manera que el aumento en el ${ }^{15} \mathrm{~N}$ y ${ }^{13} \mathrm{C}$ hacia las capas más profundas, indica la pérdida preferencial de moléculas de ${ }^{14} \mathrm{~N}$ y ${ }^{12} \mathrm{C}$ a través del fraccionamiento cinético durante la transformación biológica (Krull et al., 2006). Además, El incremento de valores de ${ }^{13} \mathrm{C}$ y ${ }^{15} \mathrm{~N}$ en función de la profundidad del suelo se atribuye también a la acumulación de biomasa microbiana (hongos saprofitos, micorrhizas arbusculares y ectomicorrhizas) (Boström et al., 2007; Hobbie y Horton, 2007; Lindahl et al., 2007; Etcheverria et al., 2009).

Teniendo en cuenta la información de los tres estratos de muestreo se encontró que el ACP explicó con dos componentes el $96,6 \%$ de la variabilidad total. El Análisis mostró por medio de vectores las correlaciones entre las variables evaluadas, dejando ver correlaciones positivas entre $\% \mathrm{C}$ y almacenamientos de C y N (sentido oeste), las cuales a su vez se correlacionaron negativamente con densidad aparente y ${ }^{15} \mathrm{~N}$ (sentido este). Los vectores de mayor longitud y más cercanos al eje horizontal hicieron parte del C1 $(88,4 \%)$, que fue el más relevante y se interpretó como el mayor contenido de carbono y nitrógeno en suelos con menor densidad aparente y abundancia de ${ }^{15} \mathrm{~N}$ (Figura 2). Los registros mostraron el efecto de la altura en el momento de proyectarse sobre el plano, agrupándose por zonas y diferenciando la parte alta $(1450-1600 \mathrm{~m})$ en función del eje horizontal, por presentar valores superiores en acumulación de materia orgánica, que fueron disminuyendo hacia las partes más bajas $(1150-1300 \mathrm{~m})$ caracterizadas por la presencia de suelos más densos y enriquecidos con el isótopo de nitrógeno.

Entre usos del terreno se encontraron algunos cambios en las abundancias ${ }^{13} \mathrm{C}$, posiblemente por la presencia de plantas $\mathrm{C}_{4}$ que demuestran que las gramíneas han comenzado a sustituir el carbono fijado en épocas anteriores por cultivos de café o relictos de selva, pero a la vez revela la capacidad que tienen las plantas $\mathrm{C}_{3}$ para estabilizar el carbono en el suelo. En algunos de los casos a pesar de haber sido reemplazadas por pastizales, han dejado fijado en el suelo un carbono recalcitrante 
Cuadro 2. Variación con la profundidad de los parámetros evaluados en las zonas de muestreo. Datos en paréntesis corresponden al error estándar.

Table 2. Depth variations of evaluated parameters in sampling zones. Data in parentheses correspond to standard error.

\begin{tabular}{|c|c|c|c|c|c|c|c|c|}
\hline $\begin{array}{l}\text { Profundidad } \\
\text { (cm) }\end{array}$ & $\mathrm{C}(\%)$ & $\begin{array}{c}N \\
(\%)\end{array}$ & $\mathrm{C}: \mathbf{N}$ & $\begin{array}{c}\mathrm{C} \\
\left(\mathrm{t} \mathrm{ha}^{-1}\right)\end{array}$ & $\begin{array}{c}\mathrm{N} \\
\left(\mathrm{t} \mathrm{ha}^{-1}\right)\end{array}$ & $\begin{array}{c}\text { DA } \\
\left(\mathrm{g} \mathrm{cm}^{-3}\right)\end{array}$ & $\begin{array}{l}\delta^{13} \mathrm{C} \\
(\%)\end{array}$ & $\begin{array}{l}\delta^{15} \mathrm{~N} \\
(\% \circ)\end{array}$ \\
\hline \multicolumn{9}{|c|}{$\bar{Z}$ Zona alta (1450-1600 m) } \\
\hline \multirow[t]{2}{*}{$0-10$} & $7.72^{\mathrm{a}}$ & $0.69^{\mathrm{a}}$ & $11.17^{\mathrm{a}}$ & $42.88^{\mathrm{a}}$ & $3.86^{\mathrm{a}}$ & $0.56^{\mathrm{a}}$ & $-24.49^{\mathrm{a}}$ & $4.59^{\mathrm{a}}$ \\
\hline & $(1.83)$ & $(0.17)$ & $(0.79)$ & $(11.60)$ & $(1.07)$ & $(0.10)$ & $(1.30)$ & $(0.83)$ \\
\hline \multirow[t]{2}{*}{$10-20$} & $6.48^{\mathrm{b}}$ & $0.56^{\mathrm{b}}$ & $11.57^{\mathrm{ab}}$ & $39.51^{\mathrm{ab}}$ & $3.43^{\mathrm{ab}}$ & $0.61^{\mathrm{b}}$ & $-24.14^{\mathrm{a}}$ & $4.92^{\mathrm{ab}}$ \\
\hline & $(1.43)$ & $(0.13)$ & $(0.97)$ & $(9.09)$ & $(0.82)$ & $(0.08)$ & $(0.98)$ & $(0.66)$ \\
\hline \multirow[t]{2}{*}{ 20-30 } & $5.75^{\mathrm{c}}$ & $0.48^{\mathrm{c}}$ & $11.97^{\mathrm{b}}$ & $37.06^{\mathrm{b}}$ & $3.12^{\mathrm{b}}$ & $0.65^{\mathrm{b}}$ & $-23.94^{\mathrm{a}}$ & $5.15^{\mathrm{b}}$ \\
\hline & $(1.25)$ & $(0.12)$ & $(1.13)$ & $(8.50)$ & $(0.78)$ & $(0.08)$ & $(0.83)$ & $(0.66)$ \\
\hline \multicolumn{9}{|c|}{ Zona media (1300-1450 m) } \\
\hline \multirow[t]{2}{*}{$0-10$} & $5.01^{\mathrm{a}}$ & $0.47^{\mathrm{a}}$ & $10.49^{\mathrm{a}}$ & $35.31^{\mathrm{a}}$ & $3.36^{\mathrm{a}}$ & $0.74^{\mathrm{a}}$ & $-24.26^{\mathrm{a}}$ & $5.63^{\mathrm{a}}$ \\
\hline & $(1.83)$ & $(0.17)$ & $(0.71)$ & $(10.97)$ & $(1.01)$ & $(0.15)$ & $(1.66)$ & $(1.41)$ \\
\hline \multirow[t]{2}{*}{$10-20$} & $3.92^{\mathrm{b}}$ & $0.36^{\mathrm{b}}$ & $10.79^{\mathrm{a}}$ & $28.37^{\mathrm{b}}$ & $2.64^{\mathrm{b}}$ & $0.74^{\mathrm{a}}$ & $-23.86^{\mathrm{b}}$ & $6.47^{\mathrm{b}}$ \\
\hline & $(1.37)$ & $(0.12)$ & $(0.98)$ & $(8.69)$ & $(0.80)$ & $(0.15)$ & $(0.99)$ & $(1.25)$ \\
\hline \multirow[t]{2}{*}{ 20-30 } & $3.41^{\mathrm{b}}$ & $0.30^{\mathrm{b}}$ & $11.15^{\mathrm{a}}$ & $24.91^{\mathrm{b}}$ & $2.25^{\mathrm{b}}$ & $0.76^{\mathrm{a}}$ & $-23.59^{\mathrm{b}}$ & $6.99^{c}$ \\
\hline & $(1.14)$ & $(0.09)$ & $(1.16)$ & $(7.23)$ & $(0.66)$ & $(0.18)$ & $(0.84)$ & $(0.94)$ \\
\hline \multicolumn{9}{|c|}{ Zona baja (1150-1300 m) } \\
\hline \multirow[t]{2}{*}{$0-10$} & $2.54^{\mathrm{a}}$ & $0.25^{\mathrm{a}}$ & $10.32^{\mathrm{ab}}$ & $24.99^{\mathrm{a}}$ & $2.43^{\mathrm{a}}$ & $0.99^{\mathrm{a}}$ & $-23.61^{\mathrm{a}}$ & $7.68^{\mathrm{a}}$ \\
\hline & $(0.57)$ & $(0.06)$ & $(0.83)$ & $(5.80)$ & $(0.56)$ & $(0.13)$ & $(1.74)$ & $(1.15)$ \\
\hline \multirow[t]{2}{*}{$10-20$} & $2.08^{\mathrm{b}}$ & $0.21^{\mathrm{b}}$ & $10.17^{\mathrm{a}}$ & $21.52^{b}$ & $2.14^{\mathrm{b}}$ & $1.03^{\mathrm{a}}$ & $-23.84^{\mathrm{a}}$ & $8.17^{\mathrm{ab}}$ \\
\hline & $(0.90)$ & $(0.09)$ & $(1.16)$ & $(10.50)$ & $(0.98)$ & $(0.13)$ & $(1.22)$ & $(1.15)$ \\
\hline \multirow[t]{2}{*}{ 20-30 } & $2.13^{\mathrm{b}}$ & $0.20^{\mathrm{b}}$ & $10.67^{\mathrm{b}}$ & $21.99^{\mathrm{b}}$ & $2.08^{\mathrm{b}}$ & $1.03^{\mathrm{a}}$ & $-23.37^{\mathrm{a}}$ & $8.29^{\mathrm{b}}$ \\
\hline & $(1.15)$ & $(0.11)$ & $(1.05)$ & (13.60) & (1.30) & $(0.14)$ & $(1.38)$ & $(1.25)$ \\
\hline
\end{tabular}

Prof: profundidad, C: carbono, N:nitrógeno, DA: densidad aparente. Letras minúsculas diferentes dentro de la misma zona indican diferencias significativas $(\mathrm{p}<0.05)$ entre profundidades. [Prof: depth, C: carbon, N: nitrogen, DA: bulk density. Different lower-case letters in the same zone, indicate significative differences $(\mathrm{p}<0.05)$ between depths]].

evidenciado en los valores de ${ }^{13} \mathrm{C}$ (entre $25,18$ y $-21,27)$ que indican que el $\mathrm{C}$ medido proviene en su gran mayoría de plantas $\mathrm{C}_{3}$ (Cuadro 3). Los relictos de selva solo se encontraron por encima de los $1300 \mathrm{~m}$ y se caracterizaron por tener suelos menos densos con bajos contenidos de $\mathrm{C}$ y ${ }^{15} \mathrm{~N}$, contrario a los pastizales que mostraron mayor densidad aparente y estuvieron enriquecidos con el isótopo de nitrógeno.

Por otra parte, monocultivos como caña panelera (Sacharun officinarum), café 


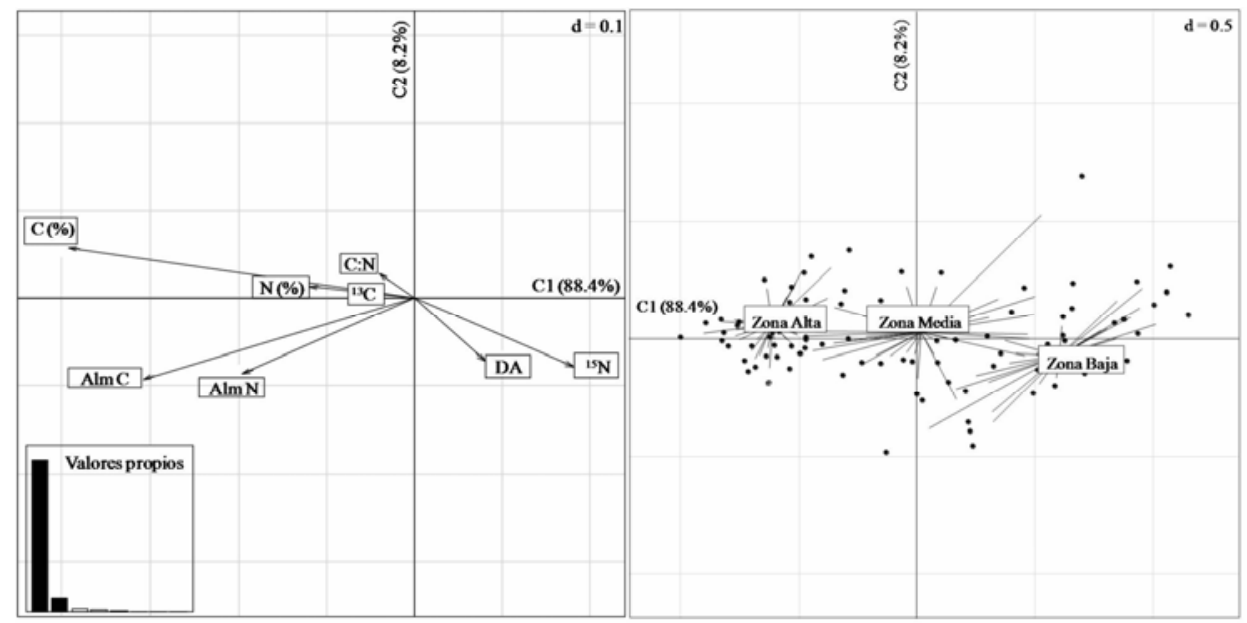

Figura 2. Correlaciones de las variables fisicoquímicas del suelo con los componentes 1 y 2 extraídos en el ACP para los tres estratos, y proyección de los sitios de muestreo en el plano, agrupados por zonas $(\mathrm{p}<0,01)$.

Figure 2. Correlations of soil physico-chemical variables with components 1 and 2 extracted in three strata PCA, and sampling sites proyection in the plane, grouped by zones $(\mathrm{p}<0.01)$.

variedad Colombia (Coffea arabica L., cv. Colombia) y caturra (Coffea arabica L., cv. caturra), con aplicaciones constantes de fertilizantes de fertilizantes de síntesis química y prácticas de manejo como podas y eliminación de arvenses, tendieron a almacenar más carbono y nitrógeno. Posiblemente, la mayor fijación en estos monocultivos se presentó porque la fertilización con nitrógeno favorece la asimilación de Amonio $\left(\mathrm{NH}_{4}{ }^{+}\right)$por parte de las plantas (Diekow et al., 2005), estimulando la producción de fitomasa que posteriormente retorna al suelo y contribuye con los depósitos de este elemento (Jones y Jacobsen, 2005).

Se encontró que para la totalidad del perfil $(0-30 \mathrm{~cm})$, los suelos presentaron ganancias en el almacenamiento de carbono cuando las coberturas vegetales naturales (relictos de selva) se transformaron en cultivos, principalmente de café y plátano, mientras que se notaron disminuciones considerables cuando los cultivos se convirtieron en pastizales. Esto muestra una tendencia contraria a la encontrada por Guo y Gifford (2002) en la que reportan pérdidas de $\mathrm{C}$ hasta del $42 \%$ cuando se transforma la selva nativa para actividades agrícolas, y ganancias hasta de un $19 \%$ cuando los cultivos se convierten en pastizales.

Además se observó que los suelos a pesar de tener el mismo uso del terreno variaron la cantidad de $\mathrm{C}$ de acuerdo con la altura, registrando disminuciones considerables como la de relicto de selva que entre la zona alta y media dejó de acumular 59,59 t C ha-1, tendencia que también se encontró en los demás usos evaluados (Cuadro 3).

Los usos con mayor grado de conservación como los relictos de selva, presentaron menores almacenamientos de C que aquellos más intensivos; posiblemente porque los relictos almacenan la mayor parte del carbono en la biomasa aérea, y al poseer una mayor estructura vertical generan diferencias en el microclima del sistema, siendo la 
Cuadro 3. Medias y desviaciones estándar (D.E.) de almacenamientos de $\mathrm{C}$ y abundancias naturales de ${ }^{13} \mathrm{C}$ en algunos usos del terreno de cada zona de muestreo $(0-30 \mathrm{~cm})$.

Table 3. Means and standar deviations of carbon stocks and ${ }^{13} \mathrm{C}$ natural abundances of some land uses in every sampling zone $(0-30 \mathrm{~cm})$.

\begin{tabular}{|c|c|c|c|c|c|}
\hline \multirow[t]{2}{*}{ Uso del terreno } & \multirow[t]{2}{*}{ n } & \multicolumn{2}{|c|}{$C\left(t_{h a}^{-1}\right)$} & \multicolumn{2}{|c|}{$\delta^{13} \mathrm{C}$ (\%o) } \\
\hline & & Media & D.E. & Media & D.E. \\
\hline \multicolumn{6}{|l|}{ Zona alta (1450-1600 m) } \\
\hline Relicto de selva & 6 & 102.67 & 15.29 & -23.74 & 1.41 \\
\hline Cafetal variedad Colombia & 8 & 120.32 & 15.41 & -23.99 & 0.79 \\
\hline Barbecho & 3 & 121.11 & 17.91 & -24.48 & 0.63 \\
\hline Café-plátano & 4 & 122.94 & 9.64 & -24.50 & 0.77 \\
\hline Pastizal & 2 & 118.01 & 5.49 & -23.79 & 0.00 \\
\hline Guadua & 3 & 130.43 & 8.51 & -25.18 & 0.52 \\
\hline Cafetal con sombrío & 2 & 125.13 & 18.79 & -24.81 & 0.06 \\
\hline Cafetal variedad caturra & 1 & 123.64 & - & -23.56 & - \\
\hline \multicolumn{6}{|l|}{ Zona media (1300-1450 m) } \\
\hline Relicto de selva & 1 & 43.08 & - & -21.27 & - \\
\hline Cafetal variedad Colombia & 6 & 101.39 & 24.49 & -24.24 & 0.53 \\
\hline Barbecho & 2 & 105.18 & 7.69 & -24.30 & 0.33 \\
\hline Café-plátano & 8 & 89.79 & 28.02 & -24.52 & 0.60 \\
\hline Pastizal & 5 & 82.52 & 22.35 & -22.85 & 1.36 \\
\hline Guadua & 1 & 87.90 & - & -24.99 & - \\
\hline Cafetal variedad caturra & 4 & 80.08 & 7.66 & -23.74 & 0.20 \\
\hline \multicolumn{6}{|l|}{ Zona baja (1150-1300 m) } \\
\hline Barbecho & 2 & 62.18 & 16.30 & -24.12 & 0.70 \\
\hline Café-plátano & 1 & 68.28 & - & -24.48 & - \\
\hline Pastizal & 12 & 64.53 & 13.28 & -23.01 & 1.0 \\
\hline Guadua & 3 & 58.24 & 15.61 & -24.88 & 0.80 \\
\hline Cafetal con sombrío & 1 & 61.58 & - & -24.96 & - \\
\hline Cafetal variedad caturra & 5 & 92.33 & 22.46 & -23.93 & 0.80 \\
\hline Caña panelera & 3 & 80.31 & 30.60 & -22.13 & 1.10 \\
\hline
\end{tabular}


atmósfera más húmeda y la temperatura relativamente baja y constante, lo que pudo haber favorecido los procesos de descomposición de materia orgánica a partir de la biota del suelo (Van der Kamp et al., 2009), reflejándose en mayores pérdidas de carbono por los procesos de respiración. Estos almacenamientos en 0$30 \mathrm{~cm}$ difirieron de otros estudios realizados en el paleo y neotrópico, en el caso de relicto de selva en la zona alta, almacenó en promedio $102,67 \mathrm{t} \mathrm{ha}^{-1}$, mientras que el mismo uso y a la misma profundidad presentó valores de $75 \mathrm{y}$ 82,20 $\mathrm{t} \mathrm{ha}^{-1}$ en los estudios de Escobar y Toriatti Dematté (1991) y Powers y Schlesinger (2002) respectivamente. Incluso, los valores fueron superiores a los obtenidos por Glenday (2006) en la profundidad 0-60 $\mathrm{cm}$ de bosques nativos en Kenia (Cuadro 4). También se observó en la mayoría de los usos evaluados una alta capacidad para el almacenamiento de $\mathrm{C}$, por lo que se puede afirmar que en general los suelos del municipio de Alcalá cuentan con un alto potencial para la captura de este elemento.

Según Lal (2004), los depósitos de carbono en el suelo y su dinámica se encuentran estrechamente relacionados con la fauna edáfica, la cual es considerada un factor de gran influencia en las propiedades físicas y biológicas del suelo, especialmente en lo relacionado con la estructura, porosidad, aireación, infiltración, drenaje, ciclaje de nutrientes y flujo de materia orgánica. Se debe resaltar el alto potencial de almacenamiento de carbono encontrado en los suelos de esta zona subandina. Al respecto Lal (2004) afirma que una de las estrategias viables para disminuir las concentraciones de $\mathrm{CO}_{2}$ en la atmósfera, es el incremento de la densidad del carbono orgánico del suelo, mejorando la distribución en profundidad y encapsulándolo dentro de los micro- -agregados, de manera que el $\mathrm{C}$ esté protegido de los procesos microbiales y se vuelva recalcitrante; lo que según Feldpausch et al. (2004) se convierte en una consideración importante para el manejo futuro de créditos de carbono. Además, los mercados se están extendiendo globalmente y los mecanismos de desarrollo limpio del protocolo de Kyoto ofrecen una oportunidad económica atractiva para la subsistencia de agricultores de países en vía de desarrollo, quiénes podrían obtener recursos económicos por medio de la venta a países industrializados del $\mathrm{C}$ secuestrado en sus agroecosistemas (Nair et al., 2009); evidenciando que la disminución de emisiones de gases de efecto invernadero a partir del almacenamiento de carbono, además de ser un servicio ecosistémico se convierte en una alternativa económica que se puede potenciar a través de las evaluaciones del carbono orgánico del suelo en paisajes rurales neotropicales.

Por lo tanto se recomienda a las instituciones encargadas del diseño de políticas relacionadas con el calentamiento global y el cambio climático (en Colombia bajo la coordinación del Ministerio del Ambiente), asignar mayor importancia a la captura de carbono en el suelo y promover su inclusión en los mercados internacionales de carbono.

Se propone para Colombia la elaboración de la línea base nacional de almacenamiento de carbono y nitrógeno en el suelo, con la que ya cuentan otros países y les ha permitido la construcción y validación de modelos, permitiendo así realizar predicciones a futuro y simular escenarios de lo que podría ocurrir con la captura de estos elementos según las características climáticas, fisiográficas y de usos y coberturas vegetales del terreno de un determinado territorio. 
Cuadro 4. Almacenamiento de carbono en diferentes usos del terreno del paleo y neotrópico.

Table 4. Carbon stock in different land uses of paleo and neotropic.

\begin{tabular}{|c|c|c|c|c|c|c|}
\hline Uso del terreno & Orden del Suelo & $\begin{array}{l}\text { Altura } \\
\text { (m) }\end{array}$ & $\begin{array}{l}\text { Prof } \\
(\mathbf{c m})\end{array}$ & $\underset{\left(t \mathrm{C} \mathrm{ha}^{-1}\right)}{\operatorname{Alm}}$ & Localización & Referencia \\
\hline Pastizal (Ischaemum sp.) & Andisol & 64 & 50 & 115.6 & Costa Rica & Jiménez et al., 2007 \\
\hline Pastizal (Zona baja) & Alfisol & $1150-1300$ & 30 & 64.53 & Colombia & Este estudio \\
\hline Pastizal & Andisol & $0-1600$ & 25 & 80.47 & Ecuador & López-Ulloa et al., 2005 \\
\hline Pastizal con árboles & Vertisol & $550-650$ & 100 & 284 & Etiopía & Michelsen et al., 2004 \\
\hline Café a libre exposición & Andisol & $1350-2150$ & 5 & 23 & Colombia & Hoyos y Comerford, 2005 \\
\hline Café variedad Colombia (Zona alta) & Andisol & $1450-1600$ & 30 & 120.32 & Colombia & Este estudio \\
\hline Café variedad caturra (Zona baja) & Alfisol & $1150-1300$ & 30 & 92.33 & Colombia & Este estudio \\
\hline Plátano & Ultisol y Alfisol & $50-300$ & 30 & 35 & Panamá & Tschakert et al., 2006 \\
\hline Barbecho (dos años) & Ultisol y Alfisol & $50-300$ & 30 & 51 & Panamá & Tschakert et al., 2006 \\
\hline Bosque secundario & Andisol & $0-1600$ & 25 & 88.50 & Ecuador & López-Ulloa et al., 2005 \\
\hline Bosque nativo (>20 años) & Acrisol y Cabrisol & $1520-1680$ & 60 & 100 & Kenia & Glenday, 2006 \\
\hline Selva húmeda tropical & Inceptisol y Ultisol & $47-799$ & 30 & 82.20 & Costa Rica & Powers y Schlesinger, 2002 \\
\hline Selva & Ultisol & & 30 & 75 & Colombia & Escobar y Toriatti Dematté, 1991 \\
\hline Relicto de selva (Zona alta) & Andisol & $1450-1600$ & 30 & 102.67 & Colombia & Este estudio \\
\hline
\end{tabular}

Prof: profundidad, Alm: almacenamiento; Prof: depth, Alm: stock 


\section{CONCLUSIONES}

Los resultados de este trabajo muestran que los almacenamientos de car nitrógeno en el suelo varían con la aııı a sobre el nivel del mar, y en el caso de Alcalá, los mayores contenidos de estos dos elementos estuvieron en la zona alta correspondiente al rango altitudinal 1450$1600 \mathrm{~m}$. Por otro lado, permitieron corroborar que los cambios en el uso del terreno afectan la captura de carbono, que está relacionada con la cobertura vegetal y las prácticas de manejo, principalmente la fertilización con productos de síntesis química.

La utilización del muestreo sistemático en forma de ventanas permitió observar la heterogeneidad del paisaje rural en Alcalá, identificando patrones de uso de acuerdo con las zonas de muestreo y la forma en que estas variaciones en los usos del terreno incidieron en las propiedades fisicoquímicas del suelo. Además, este municipio se catalogó como una zona con alto potencial para la prestación de servicios ecosistémicos relacionados con la captura de carbono, porque al comparar los resultados con los de otros estudios del paleo y neotrópico se encontró que los almacenamientos de este elemento en el suelo fueron notablemente superiores; mostrando la necesidad de la creación de la línea base nacional de almacenamiento de $\mathrm{C}$ y $\mathrm{N}$ para la planificación del territorio desde la perspectiva de la contribución a la problemática del calentamiento global.

\section{AGRADECIMIENTOS}

Los autores agradecemos a la Universidad Tecnológica de Pereira, a Colciencias y al Centro de Investigaciones y Estudios en Biodiversidad y Recursos Genéticos (CIEBREG) por financiar la realización de este trabajo. A todos los miembros del grupo Gestión en Agroecosistemas Tropicales Andinos (GATA) por su apoyo ¿urante la realización del estudio, al 'entro Internacional de Agricultura Tropical (CIAT) por su colaboración en el análisis de las muestras, y a los propietarios y administradores de los 48 predios en los que se realizaron los muestreos, por facilitarnos sus sistemas productivos.

\section{REFERENCIAS}

Alcaldía municipal de Alcalá, 2003. Esquema de Ordenamiento Territorial-Diagnóstico año 2003-2011. Secretaría de Planeación. Alcalá, Valle del Cauca, Colombia, 76 p.

Batjes, N.H. 2005. Soil carbon stocks of Jordan and projected changes upon improved management of croplands. Geoderma 132, 361371 .

Boeckx, P., Van Meirvenne, M., Raulo, F., Van Cleemput, O. 2006. Spatial patterns of $\delta^{13} \mathrm{C}$ and $\delta^{15} \mathrm{~N}$ in the urban topsoil of Gent, Belgium. Organic Geochemistry 37, 1383-1393.

Boström, B., Comstedt, D. \& Ekblad, A. 2007. Isotope fractionation and ${ }^{13} \mathrm{C}$ enrichment in soil profiles during the decomposition of soil organic matter. Oecologia, 153, 89-98.

Cenicafé (Centro Nacional de Investigaciones de Café). 2007. Unidades de suelos. Colombia. Disponible en: $\mathrm{http} / / \mathrm{www} . c e n i c a f e . o r g . c o$.

Cochran, W.G. 1974. Técnicas de muestreo. Continental S.A. México, 507 p.

Diekow, J., Mielniczuk, J., Knicker, H., Bayer, C., Dick, D.P., Ko Gel-Knabner, I. 2005. Soil C and $\mathrm{N}$ stocks as affected by cropping systems and nitrogen fertilization in a southern Brazil Acrisol managed under no-tillage for 17 years. Soil and Tillage Research 81, 87-95.

Escobar, C.J., Toriatti Dematté, J.L. 1991. Distribución de la materia orgánica y del carbono-13 en un Ultisol del piedemonte amazónico. Pasturas Tropicales 13 (2), 27-30. 
Eshetu, Z. 2004. Natural ${ }^{15} \mathrm{~N}$ abundance in soils under young-growth forests in Ethiopia. Forest Ecology and Management 187, 139-147.

Espinal, C.F., Martínez, H.J., Acevedo. X. 2005. La cadena del café en Colombia: Una mirada global de su estructura y dinámica 19912005. Ministerio de Agricultura y Desarrollo Rural. Bogotá, Colombia, 35 p. Disponible en: http:/www.agrocadenas.gov.co

Etcheverria, P., Huygens, D., Godoy, R., Borie, F. and Boeckx, P. 2009. Arbuscular mycorrhizal fungi contribute to ${ }^{13} \mathrm{C}$ and ${ }^{15} \mathrm{~N}$ enrichment of soil organic matter in forest soils. Soil Biology and Biochemistry 41, 858-861.

Feijoo, A., Zúñiga, M.C., Quintero, H., Lavelle, P. 2007. Relaciones entre el uso de la tierra y las comunidades de lombrices en la cuenca del río La Vieja, Colombia. Pastos y Forrajes 30 (2), 235-249.

Feldpausch, T.R., Rondón, M.A., Fernandes, E.C.M., Riha, S.J., Wandelli, E. 2004. Carbon and nutrient accumulation in secondary forests regenerating from degraded pastures in Central Amazônia, Brazil. Ecological Applications 14, 164-176.

Glenday, J. 2006. Carbon storage and emissions offset potential in an East African tropical rainforest. Forest Ecology and Management 235, $72-83$.

Guo, L.B., Gifford, R.M. 2002. Soil carbon stocks and land use change: a meta analysis. Global Change Biology 8, 345-360.

Hobbie, E.A., Horton, T.R. 2007. Evidence that saprotrophic fungi mobilise carbon and mycorrhizal fungi mobilise nitrogen during litter decomposition. New Phytologist 173, 447-449.

Hoyos, N., Comerford, N.B. 2005. Land use and landscape effects on aggregate stability and total carbon of Andisols from the Colombian Andes. Geoderma 129, 268-278.

IPCC (Intergovernmental Panel on Climate Change). 2007. Climate Change, 2007. Mitigation. In: B. Metz, O.R. Davidson, P.R. Bosch, R. Dave, L.A. Meyer (eds). Contribution of Working Group III to the Fourth Assessment Report of the Intergovernmental Panel on Climate Change. Cambridge University Press,
Cambridge, United Kingdom and New York, NY, USA, 30 p.

Jiménez, J.J., Lal, R., Leblanc, H.A., Russo, R.O. 2007. Soil organic carbon pool under native tree plantations in the Caribbean lowlands of Costa Rica. Forest Ecology and Management 241, 134-144.

Jones, C., Jacobsen J. 2005. Nitrogen Cycling, Testing and Fertilizer Recommendations. In: Jones, C (ed). Nutrient Management Module $\mathrm{N}^{\mathrm{o}}$. 3. Montana State University, Montana, USA, 15 p. Disponible en:

http://landresources.montana.edu/NM/Modules/ NM\%203\%20htm44493.pdf.

Krull, E.S., Skjemstad, J.O. 2003. $\delta 13 \mathrm{C}$ and $\delta^{15} \mathrm{~N}$ profiles in ${ }^{14} \mathrm{C}$-dated Oxisol and Vertisols as a function of soil chemistry and mineralogy. Geoderma 112, 1-29.

Krull, E.S., Bestland, E.A., Skjemstad, J.O., Parr, J.F. 2006. Geochemistry $\left(\delta^{13} \mathrm{C}, \delta^{15} \mathrm{~N},{ }^{13} \mathrm{C}\right.$ $\mathrm{NMR})$ and residence times $\left({ }^{14} \mathrm{C}\right.$ and OSL) of soil organic matter from red-brown earths of South Australia: Implications for soil genesis. Geoderma 132, 344-360.

Lal, R. 2004. Soil carbon sequestration to mitigate climate change. Geoderma 123, 1-22.

Leifeld, J., Bassin, S., Fuhrer, J. 2005. Carbon stocks in Swiss agricultural soils predicted by land-use, soil characteristics and altitude. Agriculture, Ecosystems and Environment 105, 255-266.

Lindahl, B.D., Ihrmark, K., Boberg, J., Trumbore, S.E., Hogberg, P., Stenlid, J., Finlay, R.D. 2007. Spatial separation of litter decomposition and mycorrhizal nitrogen uptake in a boreal forest. New Phytologist 173, 611-620.

López-Ulloa, M., Veldkamp, E., De Koning, G.H.J. 2005. Soil carbon stabilization in converted tropical pastures and forests depends on soil type. Soil Science Society of America Journal 69 (4), 1110-1117.

Michelsen, A., Andersson, M., Jensen, M., Kjøller, A., Gashew, M. 2004. Carbon stocks, soil respiration and microbial biomass in fireprone tropical grassland, woodland and forest ecosystems. Soil Biology and Biochemistry 36, 1707-1717. 
Nair, P.K.R., Nair, V.D., Kumar, B.M., Haile S.G., 2009. Soil carbon sequestration in tropical agroforestry systems: a feasibility appraisal. Environmental Science and Policy. En prensa.

Peterson, B.J., Fry, B. 1987. Stable isotopes in ecosystem studies. Annual Review of Ecological Systems 18, 293-320.

Powers, J.S., Schlesinger, W.H. 2002. Relationships among soil carbon distributions and biophysical factors at nested spatial scales in rain forests of northeastern Costa Rica. Geoderma 109, 165-190.

$R$ development core team. 2008. $R$ : A language and environment for statistical computing. $\mathrm{R}$ Foundation for Statistical Computing. Viena, Austria, 1667 p. Disponible en: http://www.Rproject.org.

SPSS, 1999. Statistical Product and Service Solution, Inc..
Tan, Z., Lal, R. 2005. Carbon sequestration potential estimates with changes in land use and tillage practice in Ohio, USA. Agriculture, Ecosystems and Environment 111, 140-152.

Tschakert, P., Coomes, O.T., Potvin, C. 2006. Indigenous livelihoods, slash-and-burn agriculture, and carbon stocks in Eastern Panama. Ecological Economics 60, 807-820.

Van der Kamp, J., Yassir, I., Buurman, P. 2009. Soil carbon changes upon secondary succession in Imperata grasslands (East Kalimantan, Indonesia). Geoderma 149, 76-83.

Zúñiga, M.C., Quintero, H., Feijoo, A. 2003. Trayectoria de los sistemas campesinos de cría en un área del piedemonte de Alcalá, Valle del Cauca. Scientia et Technica 9 (23), 117-122 